\title{
QUEEN'S
UNIVERSITY
BELFAST
}

\section{The Splitting of Double-component Active Asteroid P/2016 J1 (PANSTARRS)}

Moreno, F., Pozuelos, F. J., Novaković, B., Licandro, J., Cabrera-Lavers, A., Bolin, B., Jedicke, R., Gladman, B. J., Bannister, M. T., Gwyn, S. D. J., Vereš, P., Chambers, K., Chastel, S., Denneau, L., Flewelling, H., Huber, M., Schunová-Lilly, E., Magnier, E., Wainscoat, R., ... Micheli, M. (2017). The Splitting of Double-component Active Asteroid P/2016 J1 (PANSTARRS). Astrophysical Journal Letters, 837(1), 1-6. https://doi.org/10.3847/2041-8213/aa6036

Published in:

Astrophysical Journal Letters

Document Version:

Publisher's PDF, also known as Version of record

Queen's University Belfast - Research Portal:

Link to publication record in Queen's University Belfast Research Portal

Publisher rights

(C) 2017. The American Astronomical Society. All rights reserved.This work is made available online in accordance with the publisher's

policies. Please refer to any applicable terms of use of the publisher.

\section{General rights}

Copyright for the publications made accessible via the Queen's University Belfast Research Portal is retained by the author(s) and / or other copyright owners and it is a condition of accessing these publications that users recognise and abide by the legal requirements associated with these rights.

Take down policy

The Research Portal is Queen's institutional repository that provides access to Queen's research output. Every effort has been made to ensure that content in the Research Portal does not infringe any person's rights, or applicable UK laws. If you discover content in the Research Portal that you believe breaches copyright or violates any law, please contact openaccess@qub.ac.uk. 


\title{
The Splitting of Double-component Active Asteroid P/2016 J1 (PANSTARRS)
}

\author{
F. Moreno ${ }^{1}$, F. J. Pozuelos ${ }^{1}$, B. Novaković ${ }^{2}$, J. Licandro ${ }^{3,4}$, A. Cabrera-Lavers ${ }^{3,5}$, Bryce Bolin ${ }^{6}$, Robert Jedicke ${ }^{7}$, Brett J. Gladman ${ }^{8}$, \\ Michele T. Bannister ${ }^{9}$, Stephen D. J. Gwyn ${ }^{10}$, Peter Vereš ${ }^{11}$, Kenneth Chambers ${ }^{12}$, Serge Chastel ${ }^{12}$, Larry Denneau ${ }^{12}$, \\ Heather Flewelling ${ }^{12}$, Mark Huber ${ }^{12}$, Eva Schunová-Lilly ${ }^{12}$, Eugene Magnier ${ }^{12}$, Richard Wainscoat ${ }^{12}$, Christopher Waters ${ }^{12}$, \\ Robert Weryk $^{12}$, Davide Farnocchia ${ }^{11}$, and Marco Micheli ${ }^{13,14}$ \\ ${ }^{1}$ Instituto de Astrofísica de Andalucía, CSIC, Glorieta de la Astronomía s/n, E-18008 Granada, Spain; fernando@iaa.es \\ ${ }^{2}$ Department of Astronomy, Faculty of Mathematics, University of Belgrade, Studentski trg 16, 11000 Belgrade, Serbia \\ ${ }^{3}$ Instituto de Astrofísica de Canarias, c/Vía Láctea s/n, E-38200 La Laguna, Tenerife, Spain \\ ${ }^{4}$ Departamento de Astrofísica, Universidad de La Laguna (ULL), E-38205 La Laguna, Tenerife, Spain \\ ${ }^{5}$ GRANTECAN, Cuesta de San José s/n, E-38712, Breña Baja, La Palma, Spain \\ ${ }^{6}$ Laboratoire Lagrange, Université Côte d'Azur, Observatoire de la Côte d'Azur, CNRS, Blvd. de l'Observatoire, CS 34229, F-06304 Nice cedex 4, France \\ ${ }_{8}^{7}$ University of Hawaii, Institute for Astronomy, 2680 Woodlawn Drive, Honolulu, HI 96822, USA \\ ${ }^{8}$ Department of Physics and Astronomy, University of British Columbia, Vancouver, BC, Canada \\ ${ }^{9}$ Astrophysics Research Centre, Queens University Belfast, Belfast BT7 1NN, UK \\ ${ }^{10}$ NRC-Herzberg Astronomy and Astrophysics, National Research Council of Canada, 5071 West Saanich Road, Victoria, BC V9E 2E7, Canada \\ ${ }^{11}$ Jet Propulsion Laboratory, California Institute of Technology, 4800 Oak Grove Drive, Pasadena, CA 91109, USA \\ ${ }^{12}$ Institute for Astronomy, University of Hawaii, 2680 Woodlawn Drive, Honolulu, HI 96822, USA \\ ${ }^{13}$ SSA-NEO Coordination Centre, ESA, I-00044 Frascati (RM), Italy \\ ${ }^{14}$ INAF OAR, I-00040 Monte Porzio Catone (RM), Italy \\ Received 2017 January 3; revised 2017 February 11; accepted 2017 February 12; published 2017 February 23
}

\begin{abstract}
We present deep imaging observations, orbital dynamics, and dust-tail model analyses of the double-component asteroid P/2016 J1 (J1-A and J1-B). The observations were acquired at the Gran Telescopio Canarias (GTC) and the Canada-France-Hawaii Telescope (CFHT) from mid-March to late July of 2016. A statistical analysis of backward-in-time integrations of the orbits of a large sample of clone objects of $\mathrm{P} / 2016 \mathrm{~J} 1-\mathrm{A}$ and J1-B shows that the minimum separation between them occurred most likely $\sim 2300$ days prior to the current perihelion passage, i.e., during the previous orbit near perihelion. This closest approach was probably linked to a fragmentation event of their parent body. Monte Carlo dust-tail models show that those two components became active simultaneously $\sim 250$ days before the current perihelion, with comparable maximum loss rates of $\sim 0.7$ and $\sim 0.5 \mathrm{~kg} \mathrm{~s}^{-1}$, and total ejected masses of $8 \times 10^{6}$ and $6 \times 10^{6} \mathrm{~kg}$ for fragments $\mathrm{J} 1-\mathrm{A}$ and $\mathrm{J} 1-\mathrm{B}$, respectively. Consequently, the fragmentation event and the present dust activity are unrelated. The simultaneous activation times of the two components and the fact that the activity lasted 6-9 months or longer, strongly indicate ice sublimation as the most likely mechanism involved in the dust emission process.
\end{abstract}

Key words: methods: numerical - minor planets, asteroids: individual (P/2016 J1 (PANSTARRS))

\section{Introduction}

The double-component asteroid P/2016 J1 (PANSTARRS; components designated as J1-A and $\mathrm{J} 1-\mathrm{B}$ ) was discovered by R. Weryk and R. J. Wainscoat on CCD images acquired on May 5.5 UT with the $1.8 \mathrm{~m}$ Pan-STARRS1 telescope (Weryk \& Wainscoat 2016). The object is classified as a main-belt asteroid because its Tisserand parameter with respect to Jupiter (Kresak 1982) is $T_{J}=3.113$ (most main-belt asteroids have $T_{J}>3$ ). To date, some 20 objects in typical asteroidal orbits have been found showing transient comet-like appearance. The first object of this kind, 133P/Elst-Pizarro, was discovered in 1996, and since then it has shown alternate periods of activity and inactivity (e.g., Hsieh et al. 2004, 2010; Jewitt et al. 2014b). The orbits of these objects are found to be generally stable over timescales longer than those of Jupiter-family comets or Halley-type comets, so that they are very likely native to the asteroid belt, and not interlopers from the outer solar system (see, e.g., Haghighipour 2009; Hsieh et al. 2013).

A variety of activation mechanisms for these objects have been proposed, from impact-induced to rotational disruption (for most of the short-duration events), to ice sublimation (when the activity lasts typically a few months, in which case they are sometimes named main-belt comets). For reviews of the objects found so far, and their proposed activation mechanisms, see Bertini (2011) and Jewitt et al. (2015).

The case of $\mathrm{P} / 2016 \mathrm{~J} 1$ is remarkable as it is the first time that a double-component active asteroid sharing very similar orbital elements and patterns of activity has been discovered. In this Letter, we first report a dynamical study of the orbital evolution of the two components by backward-in-time numerical integration of their orbits in order to assess their common origin, and the fragmentation time of the parent body. And, second, we characterize the activity pattern of the two components by the photometric fit to the dust tails during the four and a half months spanned by the observations.

\section{Observations and Data Reduction}

Observations of $\mathrm{P} / 2016 \mathrm{~J} 1$ were scheduled within our Gran Telescopio Canarias (GTC) program of observations after the discovery alert. Images of P/2016 J1 have been obtained under photometric conditions on the nights of 2016 May 14, 28, and July 31 . The images were obtained on a CCD using a Sloan $r^{\prime}$ filter in the Optical System for Image and Low Resolution Integrated Spectroscopy (OSIRIS) camera-spectrograph (Cepa et al. 2000; Cepa 2010) at the GTC. The plate scale was 0 "' 254 pixel $^{-1}$. The images were bias-subtracted, flat-fielded, 


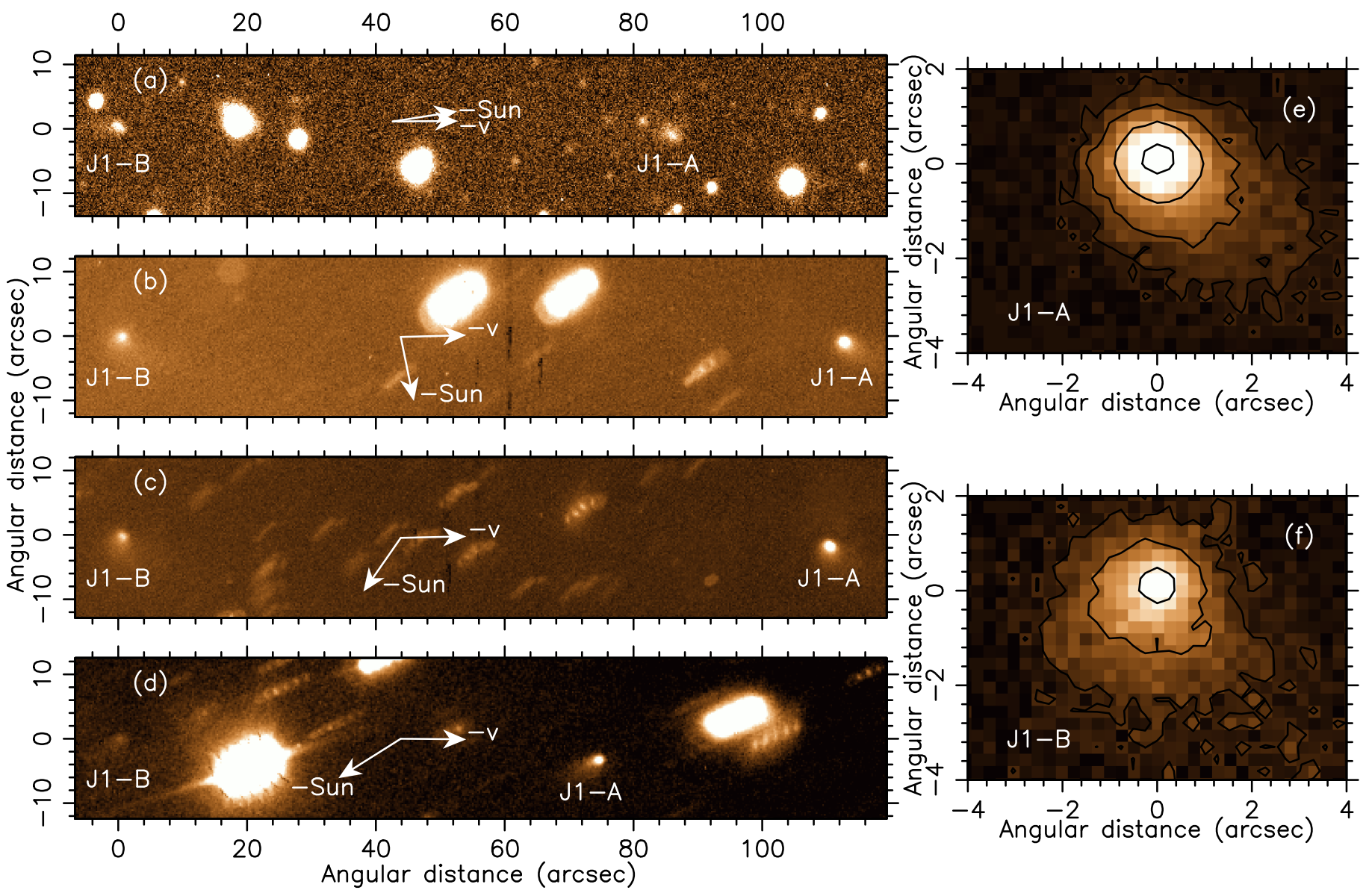

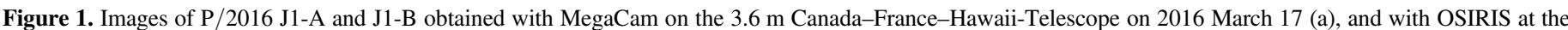

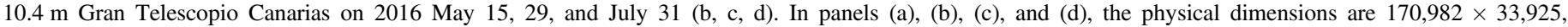

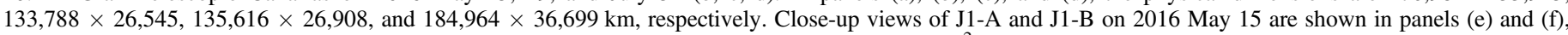

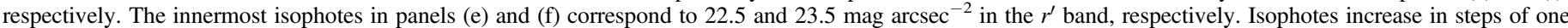

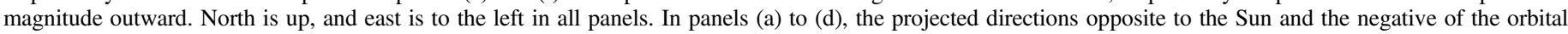
velocity vectors are shown.

and calibrated using standard stars. A median stack image was produced each night of observation from the available frames (see Figure 1).

In addition, the object was serendipitously recorded on 2016 March 17, 14:53 UT, on the MegaCam detector (Boulade et al. 2003) of the $3.6 \mathrm{~m}$ Canada-France-Hawaii Telescope (CFHT), found using Solar System Object Image Search (Gwyn et al. 2012), in an image taken as part of the Outer Solar System Origins Survey (Bannister et al. 2016). Additional data from the Pan-STARRS telescope taken between 2016 March 4 and May 5 were used to refine the orbit of and locate P/2016 J1 in the CFHT SSOS database. The MegaCam detector provides $1^{\circ}$ wide images on a mosaic of $40 \mathrm{CCDs}$ at a scale of 0 ". 184 pixel $^{-1}$. This image was obtained through the wide-band gri.MP9605 filter, using sidereal tracking, implying that the asteroid components appear trailed because of their motion on the sky (see Figure 1). Thus, it was not possible to retrieve the isophote field, although their magnitudes were determined to constrain the dust activity model.

The log of the observations is presented in Table 1. This table includes relevant geometric parameters of the observations and the magnitudes of the two components. Those magnitudes are all computed on apertures of $5000 \mathrm{~km}$ radius projected on the sky and converted to magnitudes in the the standard $R_{c}$ Cousins/Bessell band. We assume a solar-like spectrum for the scattered light from the asteroid dust. Since the asteroid is located in the outer belt, where C-type asteroids are abundant, this is consistent with the featureless, flat spectra, shown by those objects at wavelengths longer than $400 \mathrm{~nm}$ (see, e.g., de Pater \& Lissauer 2010). The conversion from Sloan- $r^{\prime}$ to $R_{c}$ magnitudes was made by $R_{c}=r^{\prime}-0.19$, obtained by assuming $\left(V-R_{c}\right)_{\odot}=0.354 \quad$ (Holmberg et al. 2006), and the photometric relation $r^{\prime}=V-0.84\left(V-R_{c}\right)+0.13$ (Fukugita et al. 1996) with $V=V_{\odot}=-26.75$ (Cox 2000). The conversion from gri. MP9605 to $R_{c}$ magnitude was performed through convolution of the solar spectrum (Neckel \& Labs 1984) with the bandpasses of the corresponding filters, resulting in $R_{c}=$ gri. MP9605+1.02.

In all cases, both asteroid components appear active at the time of the observations, so that only upper limits to the nuclear sizes can be provided. We obtain the absolute magnitudes $H$ from the $V$ magnitudes using the Bowell et al. (1989) formalism, for which we assume a slope parameter of $G=0.15$, appropriate for C-type asteroids of the outer belt. We then apply the $H$-diameter relationship by Harris \& Lagerros (2002). The highest $H$-magnitude for J1-A fragment is $H=19.22 \pm 0.12$ (2016 March 17), whereas for $\mathrm{J} 1-\mathrm{B}$ it is $H=19.35 \pm 0.03$ (2016 July 31). This would translate to maximum diameters of $\sim 1000$ and $\sim 900 \mathrm{~m}$, for J1-A and J1-B, 
Table 1

Log of the Observations

\begin{tabular}{|c|c|c|c|c|c|c|c|c|c|c|}
\hline $\begin{array}{l}\text { Observation Date (UT) } \\
\text { YYYY/MM/DD HH:MM }\end{array}$ & $\begin{array}{l}\text { Days to } \\
\text { Perihelion }\end{array}$ & $\begin{array}{c}\text { Total } \\
\text { Exposure } \\
\text { Time (s) }\end{array}$ & $\begin{array}{c}R_{c} \text {-mag } \\
\mathrm{J} 1-\mathrm{A} \\
\text { (Measured) }\end{array}$ & $\begin{array}{c}R_{c} \text {-mag } \\
\text { J1-A } \\
\text { (Model) }\end{array}$ & $\begin{array}{c}R_{c} \text {-mag } \\
\mathrm{J} 1-\mathrm{B} \\
\text { (Measured) }\end{array}$ & $\begin{array}{c}R_{c}-\mathrm{mag} \\
\mathrm{J} 1-\mathrm{B} \\
\text { (Model) }\end{array}$ & $\begin{array}{l}R^{\mathrm{a}} \\
(\mathrm{au})\end{array}$ & $\begin{array}{c}\Delta^{\mathrm{b}} \\
(\mathrm{au})\end{array}$ & $\begin{array}{l}\alpha^{\mathrm{c}} \\
\left({ }^{\circ}\right)\end{array}$ & $\begin{array}{c}\text { True } \\
\text { Anomaly } \\
\left(^{\circ}\right)\end{array}$ \\
\hline 2016 Mar 17 14:53 & -98.6 & 300 & $23.23 \pm 0.12$ & 23.23 & $23.02 \pm 0.14$ & 23.02 & 2.501 & 1.871 & 20.5 & 332.3 \\
\hline 2016 May 15 02:11 & -40.1 & 900 & $20.53 \pm 0.03$ & 20.81 & $20.80 \pm 0.04$ & 20.88 & 2.457 & 1.464 & 5.65 & 348.6 \\
\hline 2016 May 29 01:02 & -26.2 & 900 & $20.66 \pm 0.03$ & 20.73 & $20.99 \pm 0.05$ & 20.97 & 2.452 & 1.484 & 9.01 & 352.5 \\
\hline 2016 Jul 31 22:38 & +37.7 & 900 & $21.78 \pm 0.04$ & 21.85 & $23.59 \pm 0.03$ & 23.59 & 2.456 & 2.024 & 23.77 & 10.7 \\
\hline
\end{tabular}

Notes.

a Heliocentric distance.

${ }^{\mathrm{b}}$ Geocentric distance.

c Solar phase angle.

respectively, assuming a geometric albedo of $p_{v}=0.04$, appropriate for C-type asteroids. Assuming bulk densities in the 1000 to $3000 \mathrm{~kg} \mathrm{~m}^{-3}$ range this would imply escape velocities from 0.37 to $0.65 \mathrm{~m} \mathrm{~s}^{-1}$ and from 0.34 to $0.58 \mathrm{~m} \mathrm{~s}^{-1}$ for components $\mathrm{J} 1-\mathrm{A}$ and $\mathrm{J} 1-\mathrm{B}$, respectively.

\section{Orbital Dynamics Simulations}

In order to assess the probable common origin of the two asteroid components, we analyzed their possible past orbital histories. For this task we used the Orbit9 integrator embedded in the OrbFit package. ${ }^{15}$ The orbits were propagated backward in time for about 100 years (i.e., about 18 revolutions around the Sun), starting from the date of the most recent perihelion passage (2016 June 24). To explore the statistically possible orbital configurations, we generated $4 \times 10^{4}$ clone combinations drawn from the multivariate normal distribution, which is defined by the orbital covariance matrix. All the orbital data and their uncertainties are taken from the JPL Small Bodies database. The dynamical model includes as perturbing bodies all the major planets, while the clones were treated as massless particles. This analysis neglected non-gravitational perturbations, which are likely dominated by the current orbital uncertainties due to the short observed arcs used in estimating the trajectories of the two objects.

For each pair of clones, we obtained a time evolution of their mutual distances and recorded the instants of the closest approach. The results obtained for all clones are shown in the upper panel of Figure 2. They suggest two possible solutions for the age of this pair, i.e., it should be either about 900 or 2300 days old (counting from 2016 June 24).

Still, the targeting minimum distance is related to the radius of a Hill sphere that characterizes strength of the mutual gravitational interaction. For the two components studied here the Hill radius $r_{\mathrm{Hill}}$ is only about $300 \mathrm{~km}$. Therefore, to better access a possible separation date we focused on approaches within $5 r_{\text {Hill }}$, i.e., about $1500 \mathrm{~km}$.

The results taking into account only approaches within $5 r_{\mathrm{Hill}}$ are shown in the lower panel of Figure 2. There is one striking difference between the results obtained for all clones and only with those that had deep close approach. The most recent of two possible age solutions has disappeared, leaving the one about 2300 days before perihelion as the only viable option. Therefore, taking into account only close encounters observed around 2300 days before perihelion, we found a refined

\footnotetext{
15 http://adams.dm.unipi.it/orbfit/
}

estimate that separation event occurred $2300 \pm 270$ days prior to 2016 June 24.

To summarize, the obtained results strongly support the common origin of two components of $\mathrm{P} / 2016 \mathrm{~J} 1$, suggesting that a separation event likely occurred about six years ago. This implies that the current activity is not a direct consequence of the separation event.

The two components J1-A and J1-B are also a very interesting example of a population of the so-called asteroid pairs (Vokrouhlický \& Nesvorný 2009; Milani et al. 2010), being the youngest pair known so far.

\section{The Monte Carlo Dust-tail Model}

To perform a theoretical interpretation of the activity pattern associated with the asteroid components, in terms of the dust physical parameters, we used our Monte Carlo dust-tail code. This code has been used previously in several works on activated asteroids and comets, including comet 67P/Churyumov-Gerasimenko, the Rosetta target (e.g., Moreno et al. 2016c). This model computes the dust-tail brightness of a comet or activated asteroid by adding up the contribution to the brightness of each particle ejected from the parent nucleus, that, in the presence of the solar radiation pressure and gravity forces, follows a Keplerian trajectory. For a description of the code, see, e.g., Moreno et al. (2012b), Licandro et al. (2013), and Moreno et al. (2016c). The ratio of radiation pressure to the gravity force exerted on each particle is given by the parameter $\beta=C_{\mathrm{pr}} Q_{\mathrm{pr}} /(2 \rho r)$, where $C_{\mathrm{pr}}=1.19 \times 10^{-3} \mathrm{~kg} \mathrm{~m}^{-2}, Q_{\mathrm{pr}}$ is the radiation pressure coefficient, and $\rho$ is the particle density. $Q_{\mathrm{pr}}$ is taken as 1 , as it converges to that value for absorbing particles of radius $r \gtrsim 1 \mu \mathrm{m}$ (see, e.g., Moreno et al. 2012b, their Figure 5).

To make the problem tractable, a number of simplifying assumptions on the dust physical parameters must be made. Thus, the particle density is taken as $1000 \mathrm{~kg} \mathrm{~m}^{-3}$, and the geometric albedo is set to $p_{v}=0.04$, indicative of dark material of carbonaceous composition (see, e.g. Moreno et al. 2012b). For the particle phase function correction, we use a linear phase coefficient of $0.03 \mathrm{mag} \mathrm{deg}^{-1}$, which is in the range of comet dust particles in the $1^{\circ} \leqslant \alpha \leqslant 30^{\circ}$ phase angle domain (e.g., Meech \& Jewitt 1987). A broad size distribution is assumed, with minimum and maximum particle radii set to $10 \mu \mathrm{m}$ and $1 \mathrm{~cm}$, respectively, and following a power-law function of index $\kappa=-3.2$, which is in the range of previous estimates of the size distribution of particles ejected from activated asteroids and comets. 
Table 2

Best-fit Parameters of the Model for the Two Asteroid Components

\begin{tabular}{lccccr}
\hline \hline $\begin{array}{l}\text { P/2016 J1 } \\
\text { Component }\end{array}$ & $\begin{array}{c}\dot{M}_{0} \\
\left(\mathrm{~kg} \mathrm{~s}^{-1}\right)\end{array}$ & $\begin{array}{c}t_{0} \\
(\text { days })\end{array}$ & $\begin{array}{c}\text { FWHM } \\
(\text { days })\end{array}$ & $\begin{array}{c}v_{0} \\
\left(\mathrm{~cm} \mathrm{~s}^{-1}\right)\end{array}$ & $\begin{array}{c}\text { Total Dust } \\
\text { Mass Ejected (kg) }\end{array}$ \\
\hline J1-A & $0.73_{-0.10}^{+0.15}$ & $-0.8_{-5}^{+6}$ & $172_{-8}^{+11}$ & $256_{-30}^{+100}$ & $6.7_{-0.5}^{+0.5}$ \\
J1-B & $0.52_{-0.10}^{+0.10}$ & $-51.5_{-6}^{+4}$ & $132_{-7}^{+8}$ & $343_{-30}^{+40}$ & $6.7_{-0.5}^{+0.5}$ \\
\hline
\end{tabular}
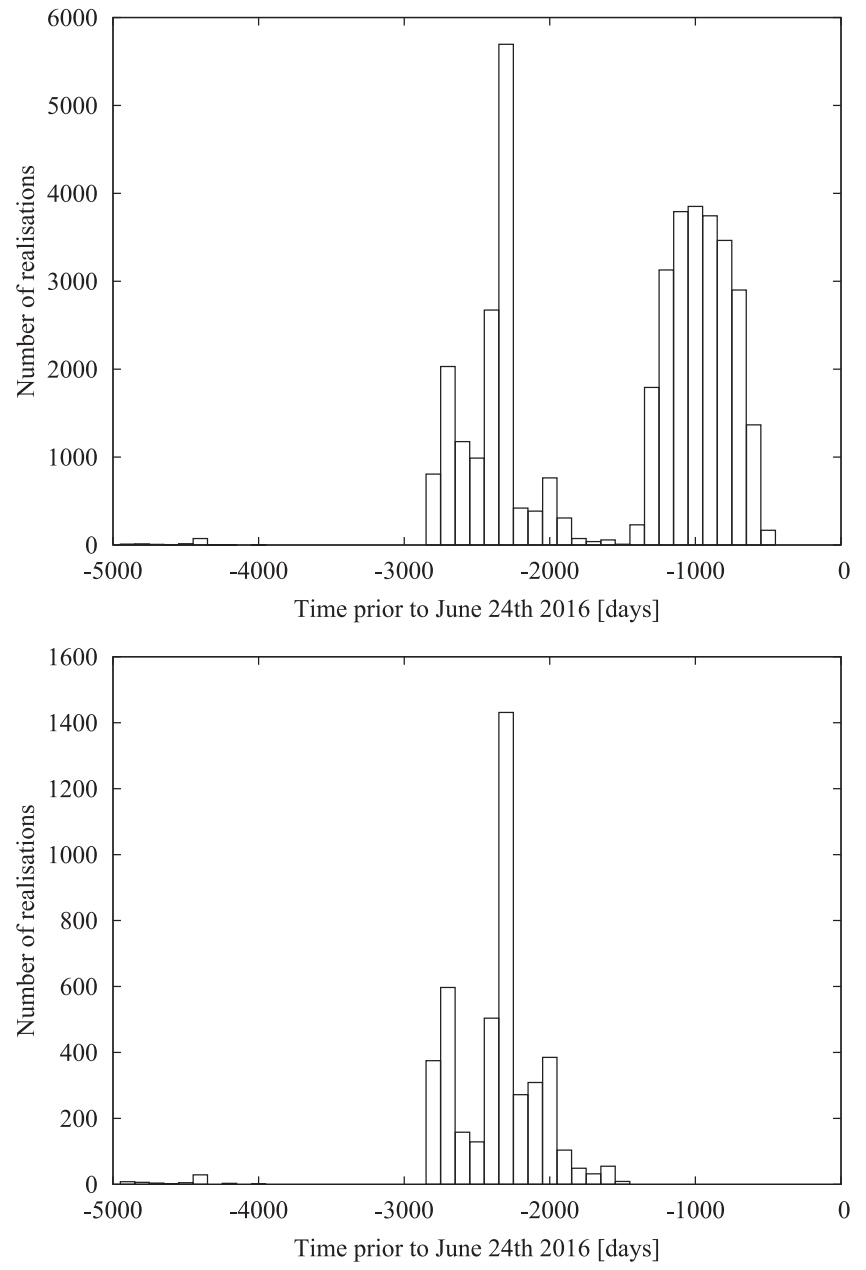

Figure 2. Upper panel: frequency distribution of dates of closest approach between J1-A and J1-B clone pairs. Lower panel: same as in the upper panel, but only the pairs of clones that approached closer than $1500 \mathrm{~km}$ are shown.

As the actual function describing the time evolution of the dust mass-loss rate is highly uncertain, we simply assume that this is given by a Gaussian function, for each component, with peak loss rate and time of maximum emission rate given by $\dot{M}_{0}$ and $t_{0}$, respectively. The FWHM of the Gaussian gives a measure of the effective time span of the emission event. This parameterization provides a fitting function with only three free parameters, which has been otherwise proved useful to characterize the behavior of other activated asteroids in the main belt (Moreno et al. 2016a, 2016b).

The particles are assumed to be ejected isotropically from the asteroid nuclei. We adopted a customary particle-size-dependent velocity law parameterized as $v=v_{0} \beta^{1 / \gamma}$, where $v_{0}$ and $\gamma$ are fitting parameters of the model. Since the ejection mechanism should be in principle the same for both fragments, we set the parameter $\gamma$ to be the same for both asteroid components.

In the modeling procedure, we have a total of nine fitting parameters: the three parameters associated to the dust-loss rate function $\left(\dot{M}_{0}, t_{0}\right.$, and HWHM), one for each component, and the dust ejection velocity parameters $v_{0}$, one for each component, and $\gamma$, this parameter being the same for both components. The model analysis, aimed at finding the best-fit set of parameters, is conducted by the downhill simplex method (Nelder \& Mead 1965), using the FORTRAN implementation described in Press et al. (1992). The quality of the fits is characterized by minimizing the mean relative error of each model image as $\sigma_{i}=\frac{\sum \frac{\left|\log \left(I_{\mathrm{obs}}(i)\right)-\log \left(I_{\bmod }(i)\right)\right|}{\mid \log \left(I_{\mathrm{obs}}(i) \mid\right.}}{N(i)}$, where $I_{\mathrm{obs}}(i)$ and $I_{\text {mod }}(i)$ are the observed and modeled tail brightness, and $N(i)$ is the number of pixels of image $i$. For the images in which the isophote field could not be retrieved, this parameter is calculated as $\sigma_{i}=\sum \frac{\left|m_{\mathrm{obs}}(i)-m_{\mathrm{mod}}(i)\right|}{m_{\mathrm{obs}}(i)}$, where $m_{\mathrm{obs}}(i)$ and $m_{\mathrm{mod}}(i)$ are the measured and modeled magnitudes. The fitting parameter is $\chi=\sum \sigma_{i}$, where the summation is extended to all the images under consideration, i.e., $i=1,8$.

\section{Results and Discussion}

The fitting of the images was accomplished by defining a set of five parameters per asteroid component, as stated in the previous section. The best-fit parameters, after running the code for a variety of different starting simplexes, are shown in Table 2 . The derived synthetic $R_{c}$ magnitudes for each image are given in Table 1 , together with the measured values. The resulting modeled isophotes are displayed in Figure 3. The agreement between the observations and the model isophotes and between the measured and synthetic magnitudes is very good, the mean of the absolute differences being only 0.07 mag. The uncertainties in the determination of the best-fit parameters are calculated assuming a criterion for which a fit is not acceptable when $\chi$ exceeds $10 \%$ of its best-fit value $(\chi=0.069)$.

From the results obtained, we see that both fragments became active before perihelion. The activation times are very similar, $\sim-250$ days to perihelion. The activity peak occurs very close to perihelion for the J1-A component, but nearly two months before for the J1-B component. In both cases, the activity lasted several months, a typical behavior of main-belt comets. The integrated ejected dust masses until the last observation of 2016 July 31 are similar, with values of $(8 \pm 2) \times 10^{6} \mathrm{~kg}$ and $(6 \pm 2) \times 10^{6} \mathrm{~kg}$ for $\mathrm{J} 1-\mathrm{A}$ and $\mathrm{J} 1-\mathrm{B}$, respectively.

Combining the orbit dynamics results with the modeled activity, the most likely scenario is that of a fragmentation event during the previous asteroid orbit, whose fragments have become activated when near perihelion in the current orbit. The simultaneous activation times for both components and the duration of the activity, of at least 6-9 months, implies almost unambiguously that ice sublimation is the mechanism 

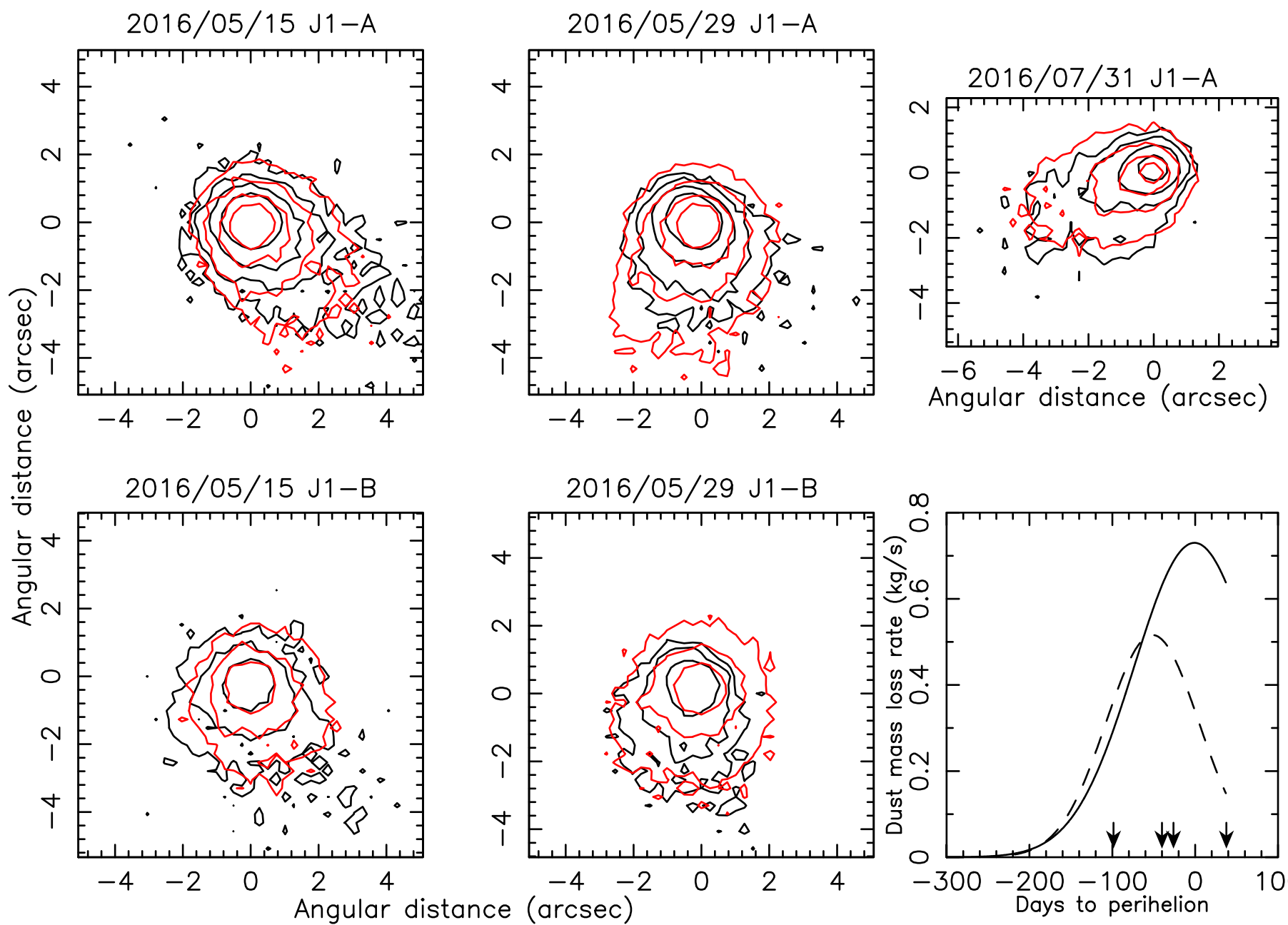

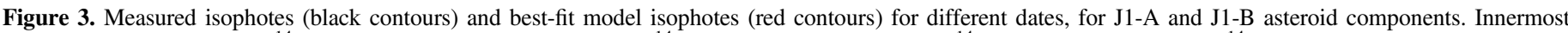

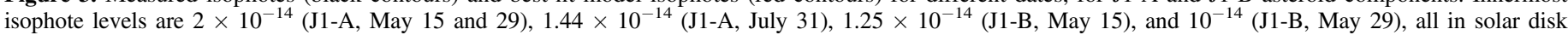

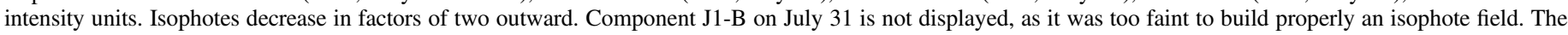

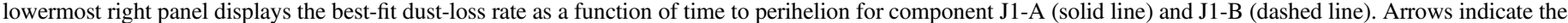
observation dates (see Table 1).

responsible for the dust emission. Archival image search for the asteroid appearance during the previous orbit, in particular during the perihelion passage, would be needed to confirm the fragmentation event.

\section{Conclusions}

From the observations of this double-component, outer main-belt, asteroid P/2016 J1-A and J1-B, its orbital dynamics, and the dust-tail modeling, the following conclusions can be drawn:

(1) The orbital dynamics computations on a large number $\left(4 \times 10^{4}\right)$ of clone asteroids, randomly chosen from the respective six-dimensional uncertainty ellipsoid in the orbital element space around each of the nominal orbits of P/2016 J1A and J1-B components, reveal a fragmentation event that most likely occurred $\sim 2300$ days before the current orbit's perihelion. Thus, $\mathrm{P} / 2016 \mathrm{~J} 1$ is a very remarkable case of an asteroid pair, being the youngest discovered so far.

(2) From dust-tail modeling, we conclude that both components become active at nearly the same time: $\sim 250$ days before perihelion passage. Both components display different evolution, with peak emission rates at different times (near perihelion and $\sim 50$ days to perihelion for J1-A and J1-B) and total dust ejected of $(8 \pm 2) \times 10^{6} \mathrm{~kg}$, and $(6 \pm 2) \times 10^{6} \mathrm{~kg}$, respectively, until the latest observation of 2016 July 31.

(3) The dust velocity parameters inferred are very similar for both asteroidal components, with terminal velocities weakly dependent on the particle size and of order $0.6-0.9 \mathrm{~m}^{-1}$ for the largest particles ejected in the model. This is compatible with the escape velocities expected from the maximum $\sim 500 \mathrm{~m}$ radius bodies in the 1000 to $3000 \mathrm{~kg} \mathrm{~m}^{-3}$ bulk density range inferred from their absolute magnitudes, assuming a geometric albedo of 0.04 .

(4) The most probable time of the closest approach between components and the start of the current dust activity are separated approximately by one orbital period. Then, the most plausible scenario is that of a fragmentation of the parent asteroid in the previous orbit, whose fragments have become activated nearly simultaneously in the present perihelion approach. This, together with the long-standing activity (6-9 months or longer), strongly suggests ice sublimation as the responsible mechanism of the dust emission.

We are grateful to an anonymous referee for comments and suggestions that helped to improve the Letter considerably. 
This Letter is based on observations made with the Gran Telescopio Canarias, installed in the Spanish Observatorio del Roque de los Muchachos of the Instituto de Astrofísica de Canarias, in the island of La Palma, and on observations obtained with MegaPrime/MegaCam, a joint project of CFHT and CEA/IRFU, at the Canada-France-Hawaii Telescope (CFHT), which is operated by the National Research Council (NRC) of Canada, the Institut National des Science de l'Univers of the Centre National de la Recherche Scientifique (CNRS) of France, and the University of Hawaii. This work is based in part on data products produced at Terapix available at the Canadian Astronomy Data Centre as part of the CanadaFrance-Hawaii Telescope Legacy Survey, a collaborative project of NRC and CNRS.

This work was supported by contracts AYA2015-67152-R and AYA2015-71975-REDT from the Spanish Ministerio de Economía y Competitividad (MINECO). J.L. gratefully acknowledges support from contract AYA2015-67772-R (MINECO, Spain).

The Pan-STARRS1 Surveys (PS1) have been made possible through contributions of the Institute for Astronomy, the University of Hawaii, the Pan-STARRS Project Office, the Max-Planck Society and its participating institutes, the Max Planck Institute for Astronomy, Heidelberg and the Max Planck Institute for Extraterrestrial Physics, Garching, The Johns Hopkins University, Durham University, the University of Edinburgh, Queen's University Belfast, the HarvardSmithsonian Center for Astrophysics, the Las Cumbres Observatory Global Telescope Network Incorporated, the National Central University of Taiwan, the Space Telescope Science Institute, the National Aeronautics and Space Administration under grant Nos. NNX08AR22G, NNX12AR65G, and NNX14AM74G issued through the Planetary Science Division of the NASA Science Mission Directorate, the National Science Foundation under grant No. AST-1238877, the University of Maryland, and Eotvos Lorand University (ELTE), and the Los Alamos National Laboratory.

B.N. also acknowledges support by the Ministry of Education, Science and Technological Development of the Republic of Serbia, Project 176011.
B.T.B. is supported by l'Ècole Doctorale Sciences Fondamentales et Appliquées, ED.SFA (ED 364) at l'Université de Côte d'Azur.

D.F. conducted this research at the Jet Propulsion Laboratory, California Institute of Technology, under a contract with NASA.

\section{References}

Bannister, M. T., Kavelaars, J. J., Petit, J.-M., et al. 2016, AJ, 152, 70 Bertini, I. 2011, P\&SS, 59, 365

Boulade, O., Charlot, X., Abbon, P., et al. 2003, Proc. SPIE, 4841, 72 Bowell, E., Hapke, B., Domingue, D., et al. 1989, in Asteroids II, ed. R. P. Binzel, T. Gehrels, \& M. S. Matthews (Tucson, AZ: Univ. Arizona Press), 524

Cepa, J. 2010, in Highlights of Spanish Astrophysics V (Berlin: Springer), 15 Cepa, J., Aguiar, M., Escalera, V., et al. 2000, Proc. SPIE, 4008, 623 Cox, A. N. 2000, Allens Astrophysical Quantities (4th ed.; Berlin: Springer) de Pater, I., \& Lissauer, J. J. 2010, Planetary Sciences (Cambridge: Cambridge Univ. Press)

Fukugita, M., Ichikawa, T., Gunn, J. E., et al. 1996, AJ, 111, 1748

Gwyn, S. D. J., Hill, N., \& Kavelaars, J. J. 2012, PASP, 124, 579

Haghighipour, N. 2009, M\&PS, 44, 1863

Harris, A. W., \& Lagerros, J. S. V. 2002, in Asteroids III, ed. W. F. Bottke et al. (Tucson, AZ: Univ. Arizona Press), 205

Holmberg, J., Flynn, C., \& Portinari, L. 2006, MNRAS, 367, 449

Hsieh, H. H., Jewitt, D., \& Fernández, Y. 2004, AJ, 127, 299

Hsieh, H. H., Jewitt, D., Lacerda, P., et al. 2010, MNRAS, 403, 363

Hsieh, H. H., Kaluna, H. M., Novaković, B., et al. 2013, ApJL, 771, L1

Jewitt, D., Agarwal, J., \& Hsieh, H. 2015, Asteroids IV (Tucson, AZ: Univ. Arizona Press)

Jewitt, D., Masateru, I., Weaver, H., et al. 2014b, AJ, 147, 117

Kresak, L. 1982, BAICz, 33, 104

Licandro, J., Moreno, F., de León, J., et al. 2013, A\&A, 550, 17

Meech, K. J., \& Jewitt, D. C. 1987, A\&A, 187, 585

Milani, A., Knežević, Z., Novaković, B., \& Cellino, A. 2010, Icar, 207, 769

Moreno, F., Licandro, J., Cabrera-Lavers, A., et al. 2016a, ApJ, 826, 137

Moreno, F., Licandro, J., Cabrera-Lavers, A., et al. 2016b, ApJL, 826, L22

Moreno, F., Pozuelos, F., Aceituno, F., et al. 2012b, ApJ, 752, 136

Moreno, F., Snodgrass, C., Hainaut, O., et al. 2016c, A\&A, 587, A155

Neckel, H., \& Labs, D. 1984, SoPh, 90, 205

Nelder, J. A., \& Mead, R. 1965, CompJ, 7, 308

Press, W. H., Teukolsky, S. A., Vetterling, W. T., \& Flannery, B. P. 1992, Numerical Recipes in FORTRAN (Cambridge: Cambridge Univ. Press)

Vokrouhlický, D., \& Nesvorný, D. 2009, AJ, 137, 111

Weryk, R., \& Wainscoat, R. 2016, CBET, 4276 